\title{
Resistive switching observation in a gallium-based liquid metal/graphene junction
}

Diego Gutiérrez, ${ }^{\ddagger a}$ Jesús Alejandro de Sousa, ${ }^{\ddagger a b}$ Marta Mas-Torrent ${ }^{a}$ and Núria Crivillers ${ }^{* a}$

anstitut de Ciència de Materials de Barcelona (ICMAB-CSIC), Campus de la UAB s/n, Bellaterra, 081093, Spain

bLaboratorio de Electroquímica, Departamento de Química, Facultad de Ciencias, Universidad de los Andes, 5101 Mérida, Venezuela

ABSTRACT. Resistive switching effect is observed for a gallium-indium/gallium oxide/graphene junction. The use of a gallium-based liquid metal alloy, in this case, the eutectic gallium-indium with its native gallium oxide skin, directly provides the metal top contact and the oxide layer needed to fabricate a memory. Graphene is used as bottom electrode due to its electrical properties and, importantly, because it prevents the formation of alloys, leading to a stable simple junction. With this structure, the ON/OFF ratio at $0.5 \mathrm{~V}$ between the high resistance state (HRS) and low resistance state (LRS) reached is $\sim 10^{4}$ in ambient conditions. The deposition of an additional switching layer is not needed compared to other resistive random access memories, which makes this system less complex to fabricate. The migration of the oxygen atoms of the oxide layer would 
be intuitively considered the main reason for the modulation of the tunneling junction resistance but, we suggest that this is not the case and instead of that, charge-trapping/detrapping at the very interface may dominate the switching function.

KEYWORDS: liquid metal, eutectic gallium-indium, gallium oxide, graphene, resistive switching

\section{INTRODUCTION}

Beyond transistor and capacitor-type memories, there are the resistor-type memories, in which data is stored by different electrical conductivity states (i.e. between a high resistance state (HRS) and a low resistance state (LRS)). These type of memories are very appealing because of their simple memory structure: a switching layer sandwiched between two electrodes. ${ }^{1}$ These electrical states are set (write) by applying a relative high voltage $\left(\mathrm{V}_{\mathrm{W}}\right)$ and, to read the conductive states a relative low voltage $\left(V_{R}\right)$ is implemented, always fulfilling $\left|V_{R}\right|<\left|V_{W}\right|$. In this way, resistive switching-based memories overcome the problem of write-after-read process. It is worth mentioning that resistive random access memories are attracting much attention because they are able to imitate the synaptic functions, playing a key role in emulating the neuromorphic computing system. ${ }^{2}$ Various mechanisms have been proposed to explain electrical conductance in resistive switching-based memories. ${ }^{3}$ For metal oxide based resistive random access memory [RRAM] (i.e., metal-insulator-metal (MIM) junctions), conducting filament type (which considers the migration of metallic cations and oxygen vacancies) and interface type (dealing mainly with interface barrier height between the electrode and the switching layer or interface defects between each layer) have been described as the two main mechanisms governing the switching phenomenon. ${ }^{4}$ Recent examples have shown the use of graphene oxide as the switching layer, in which filamentary formation, ${ }^{5}$ oxygen migration ${ }^{6}$ and redox reactions at the interfacial region between the titanium 
top electrode and the graphene oxide ${ }^{7}$ were demonstrated. In this regard, metals having high affinity to oxygen, such as aluminum or titanium, seem very attractive for resistive switching due to the possibility to create an interfacial region. ${ }^{7,8}$

In this work, as an alternative to those metals, we suggest the use of a gallium-based liquid metal (LM) alloy, the eutectic-gallium indium alloy (EGaIn). Interestingly, the gallium and galliumbased alloys have attracted not only attention for electronics, nanotechnology, mechanical engineering, energy, catalysis but also for biomedical applications because its bio-compatibility. ${ }^{9-}$ ${ }^{12}$ The EGaIn is a low-viscosity liquid at room temperature and possesses unique properties such as inherent flexibility/stretchability, high electrical and thermal conductivity, it forms alloys readily with most metals, it is non-toxic and it has a thin passivating oxide skin that provides its non-Newtonian character. ${ }^{13,14}$ The thickness of the GaOx, spontaneously formed in air has been reported to be about $0.7 \mathrm{~nm} .{ }^{15}$ One likely mechanism that has been proposed to govern the oxide growth on gallium alloys is based on the Cabrera-Mott oxidation model which was developed for solid metals. ${ }^{16}$ This mechanism is explained considering that, in spite of the thin oxide layer formed at the beginning of the exposure of the EGaIn to air, electrons from the GaIn core can tunnel through this skin layer and a chemical reduction of the adsorbed oxygen can take place. This process results in the formation of an electrostatic potential between the oxide-air and oxide-metal interfaces called Mott potential ( $\mathrm{V}_{\mathrm{M}}$ ). The resultant electric field, due to $\mathrm{V}_{\mathrm{M}}$, can become large enough to drive the diffusion of ions through the oxide, facilitating the oxide growth. ${ }^{10}$ Interestingly for the purpose of this work, the gallium oxide is currently considered a very promising material for RRAM. ${ }^{17,18}$ For example, a non-filamentary memristive behavior was shown for an amorphous gallium oxide thin film embedded between two electrodes (Pt and ITO). The reason for the switching was determined to be the bulk oxide ion conductivity within the oxide 
film, i.e. a modulation of the oxygen concentration profile as a result of the electrochemical polarization due to the applied voltage. ${ }^{17}$

Thanks to the poor alloy formation with $\mathrm{sp}^{2}$-carbon materials, the contact between gallium LM alloy and graphene have been shown to be an attractive strategy to stabilize these type of electrical contacts expanding their potential use. ${ }^{13}$ For this reason, and for the extraordinary properties of graphene-based electrodes, ${ }^{19}$ this has been the choice as a bottom electrode in the herein studied junction. The development of the chemical vapor deposition technique (CVD),${ }^{20}$ the chemical reduction of graphene oxide ${ }^{21,22}$ and the several methodologies established to transfer CVD graphene to transparent substrates, ${ }^{23,24}$ have promoted the use of graphene for several technological applications, especially because its record value of charge mobility is not inferior to the best values found in cleaved graphene. ${ }^{25}$

In this work, the electrical properties of the junction: $\mathrm{EGaIn} / / \mathrm{GaO}_{\mathrm{x}}$ /transferred $^{\mathrm{CVD}}$ graphene monolayer/quartz $\left({ }^{t} \mathrm{Gr}-\mathrm{q}\right)$ and, its potential for resistive switching applications in ambient conditions was investigated and demonstrated. For that, CVD-graphene grown on copper transferred onto quartz via wet chemistry was top-contacted with a $\mathrm{EGaIn} / \mathrm{GaO}$ x electrode coneshaped. With this structure, an ON/OFF ratio at $0.5 \mathrm{~V}$ between the high resistance state (HRS) and low resistance state (LRS) reached $\sim 10^{4}$.

\section{EXPERIMENTAL SECTION}

Carbon Substrates: All the graphene substrates employed in this work were purchased from Graphenea (Spain). The transferred graphene on quartz and $\mathrm{SiO}_{2}$, was first grown by CVD on copper foil and then transferred to a $10 \times 10 \mathrm{~mm}^{2}$ substrate of quartz (500 $\mu \mathrm{m}$ thickness) or $\mathrm{SiO}_{2}$ by a wet transfer process. Single-layer graphene grown on copper foil and PMMA (60nm) on CVD 
graphene on copper substrates were purchased from the same company. The transfer process onto different substrates (gold and glass) was done following the well stablished protocol. ${ }^{26}$ Highly pyrolytic graphite (HOPG grade ZYB) was purchase from Ted Pella INC and used after being freshly cleaved.

Junction formation and electrical measurements: EGaIn/GaOx/substrate junctions were fabricated by placing in contact a $\mathrm{EGaIn} / \mathrm{GaO}_{\mathrm{x}}$ drop hanging from a Hamilton syringe. The drop is molded in the form of cone with a tip of approximately $20 \mu \mathrm{m}$ of diameter following a previous reported procedure, without further modifications on the protocol. ${ }^{27}$ The protocol for the statistical analysis is described in the supporting information.

The EGaIn cone, working as top-electrode, was biased while the bottom electrode was grounded. An electrometer Keithley 2604B was used to perform the electrical measurements. The current density versus potential curves (JV) were obtained with an integration time settle between 50msec to 250 msec depending on the current value and a waiting time between two consecutive potential values of typically $100 \mathrm{msec}(100 \mathrm{mV} / 100 \mathrm{msec})$.

Scanning Electron Microscopy: SEM images were acquired with a FEI Magellan 400L XHRSEM.

\section{RESULTS AND DISCUSSION}

In the used customized-built experimental set-up (see Scheme S1 in the supporting information), the fabrication of the resistive switching system at ambient conditions required a first initial activation step. This consisted in a very simple procedure. First, at a given point of the sample, the freshly prepared EGaIn/ $\mathrm{GaO}_{x}$ cone-shaped $\mathrm{e}^{27}$ was placed in contact with the ${ }^{t} \mathrm{Gr}$-q surface, with a geometrical contact diameter around $20 \mu \mathrm{m}$. This first generated junction led to a $J V$ curve characteristic of an ohmic contact between the two electrodes (when sweeping the voltage in the 
range $\pm 0.5 \mathrm{~V}$ ) (Figure 1a). Secondly, the tip was retracted from the sample with the micromanipulator for a few seconds and, contacted again in the same position. Then, a new higher tunneling resistance interface was generated with a notable non-ohmic behavior (Figure 1b), indicating the formation of an insulating oxide layer. When this process was done in a wider bias window $( \pm 2 \mathrm{~V})$ a complete generation of the passivation layer was achieved (Figure 2). The same electrical response was observed for a junction based on CVD monolayer graphene grown on copper (EGaIn/GaOx $/ \mathrm{Gr}-\mathrm{Cu}$ ) (Figure S1). As an alternative to this method, the oxide passivation layer was formed by exposing the fresh-fabricated tip to ambient atmosphere. To explore the required time, two sets of experiments were carried out. First, after acquiring the $J V$ curves with a freshly prepared tip, this was exposed to air for 20 minutes, measuring $J$ in intervals of 5 minutes in the $\pm 0.5 \mathrm{~V}$ voltage range. As expected, $J$ decreased abruptly after 5 minutes and then, we only observed slight changes during 15 minutes (Figure S2a). On the contrary, Figure S2b shows that when a new tip is placed in contact with the substrate and kept in contact for 20 minutes, there is only a minor change of the measured $J$. This indicates that the tip oxidation takes place at much slower rate when it is not retracted from the surface. Furthermore, freshly prepared tips have been exposed to different atmospheres (air, argon, oxygen enriched atmosphere and higher humidity). It was observed that under argon, the measured $J$ hardly changes after 20 minutes of exposure while under oxygen and high humidity (70\%) environment the tendency is very similar to the one observed under air (Figure S3). As described later in the manuscript, both approaches led to the formation of the required interface for the resistive switching junction formation.

Additionally, the graphene layer was also investigated after the formation of the junction with the EGaIn/ $\mathrm{GaO}_{\mathrm{x}}$ electrode. For this, the XPS analysis of the $\mathrm{C} 1 \mathrm{~s}$ region was performed on a ${ }^{\mathrm{t}} \mathrm{Gr}-\mathrm{q}$ surface before and after the electrical measurements (Figure S4). The major difference between 
before and after is the $\mathrm{C}_{\mathrm{sp} 2} / \mathrm{C}_{\mathrm{sp} 3}$ ratio, which is 3.24 and 1.42 , respectively. This is attributed to unintentional hydrocarbon contamination from ambient air due to the time exposure of the substrate that is analyzed in XPS chamber upon being exposed for several hours to ambient air. ${ }^{28}$ The $\mathrm{Cc}_{\mathrm{o}} / \mathrm{C}_{\mathrm{c}=\mathrm{O}}$ is almost the same, being 3.27 for the $\mathrm{t}-\mathrm{Gr} / \mathrm{q}$ as received and 2.92 after the electrical measurements. Hence, these values point that the graphene substrate is not significantly modified (for example to partially oxidized graphene) due to the junction formation.

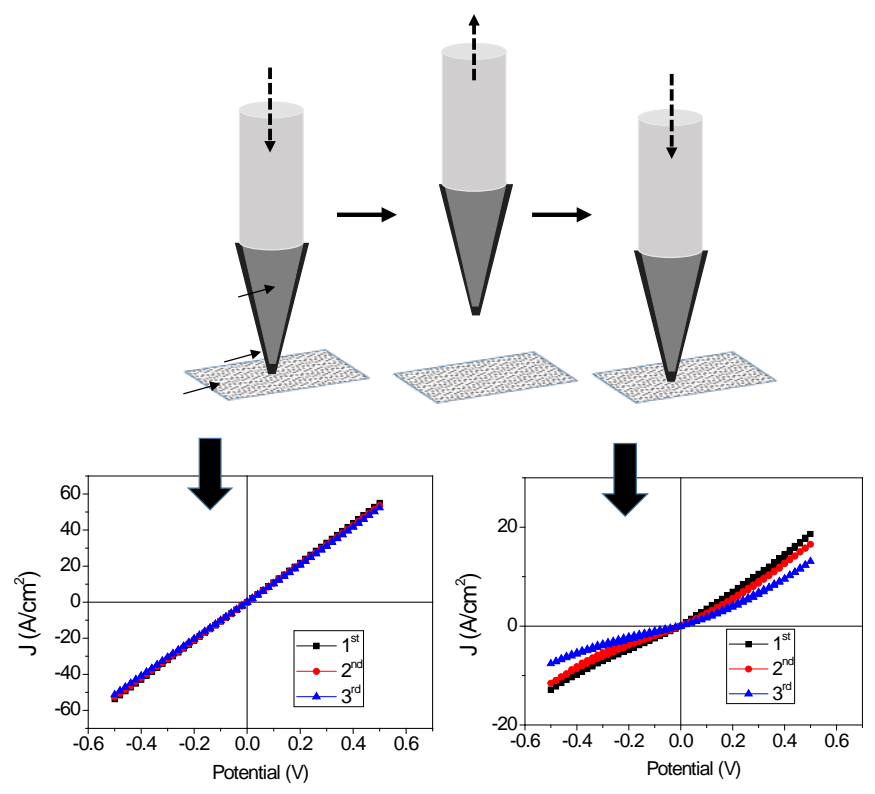

Figure 1. Scheme of the tip movement and the corresponding $J V$ curves acquired in the $\pm 0.5 \mathrm{~V}$ range for: a) a fresh EGaIn/GaOx/ $/{ }^{t} \mathrm{Gr}-\mathrm{q}$ junction, b) upon retracting and contacting the tip again.

Having all this in mind, the $\mathrm{EGaIn} / \mathrm{GaO}_{\mathrm{x}} /{ }^{t} \mathrm{Gr}-\mathrm{q}$ junctions were explored as memory units. For this, first a potential ramp from $0 \mathrm{~V}$ to $+2.2 \mathrm{~V}$ to $-2.2 \mathrm{~V}$ to $0 \mathrm{~V}$ and repeated 3 times was applied for a freshly prepared $\mathrm{EGaIn} / \mathrm{GaO}_{\mathrm{x}}$ cone. As before, a non-hysteretic behavior was observed (Figure 2a). Then, the EGaIn/GaOx cone was retracted for a few seconds (around 3 seconds) and contacted again at the same point on the graphene substrate. At this point, the application of a $\pm 2.2 \mathrm{~V}$ cycle showed hysteretic $J V$ curves as a very stable electrical output of the junction (Figure 2b). As shown 
in Figure S5, the same switching performance was achieved when the fresh prepared tip was exposed to ambient atmosphere for 20 minutes without the tip retracting step. As observed in Figure $2 \mathrm{~b}$, when the potential was swept from $0 \mathrm{~V}$ to $+2.2 \mathrm{~V}$ the junction switched to a low resistance state (LRS). The LRS was preserved even at negative potential values but once the voltage reached lower potential than $-1 \mathrm{~V}, J$ suddenly decreased approximately 2 orders of magnitude, which was identified as the high resistance state (HRS). As seen in Figure S6, the junction shows a bipolar abrupt switch. ${ }^{3}$ In several junctions the sweep potential was inverted (0V to $-2.2 \mathrm{~V}$ to $+2.2 \mathrm{~V}$ to $0 \mathrm{~V}$ ) demonstrating that the LRS was always obtained at positive potential (higher than $1 \mathrm{~V}$ ) and the HRS at negative potential values (lower than -1V) (Figure S7), regardless of the initial sweep direction. The initial state of the junctions was always the HRS. It is also important to mention that for larger geometrical contact areas (from $\varnothing_{\text {tip }} \sim 28 \mu \mathrm{m}$ to $37 \mu \mathrm{m}$ ), the memory behavior was not observed and, instead the junction showed an Ohmic contact. The hysteretic behavior was recovered when the area was diminished (Figure S8). We believe that the roughness of the tip, demonstrated by SEM images of an "inverted tip"15 (Figure S9), is responsible for such ill-defined interface which makes the junction very sensitive to small changes in the formal contact area. Interestingly, this can be slightly improved, when the measurements are performed under an oxygen richer atmosphere. In this case, the memory remains stable regardless the increase of the geometrical contact area up to $\varnothing_{\text {tip }} \sim 50 \mu \mathrm{m}$ (Figure S10), attributed to a more homogenous degree of oxidation of the Ga exposed on the tip surface. In the case of measurements of molecular self-assembled monolayers on metal substrates through EGaIn contacts, it has been shown that for large junctions, the leakage current dominates the measured current, contrary to the molecular effect which dominates in small junctions. ${ }^{15}$ As expected, this remarkable reversible resistive switching, was also observed on commercially available transferred CVD graphene on 
$\mathrm{SiO}_{2}$ and on non-commercial CVD graphene transferred onto a glass slide (see experimental section and Figure S11).
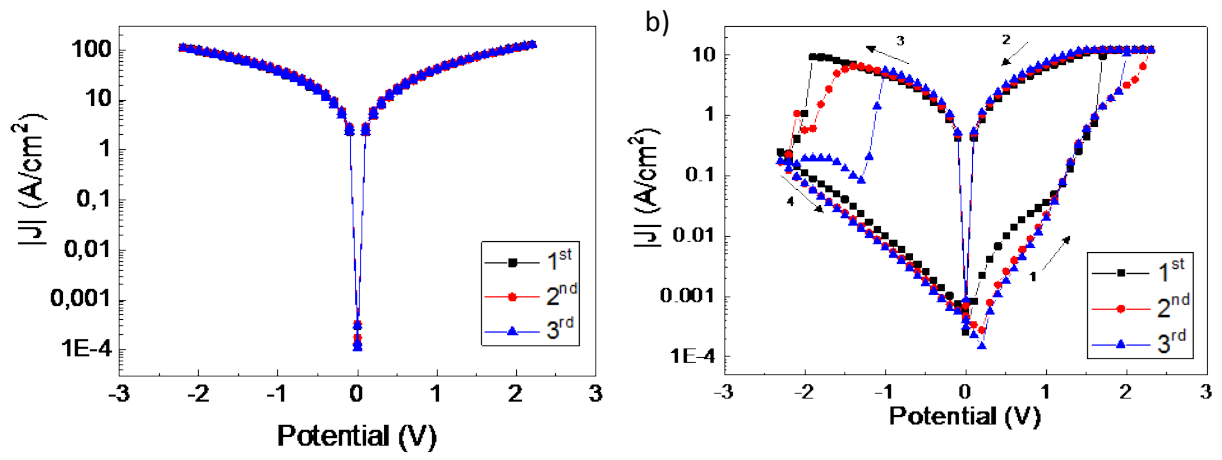

Figure 2. Exemplary $J V$ curves in a given junction. a) $J V$ curves acquired in the $\pm 2 \mathrm{~V}$ range for a junction fabricated with a freshly prepared $\mathrm{EGaIn} / \mathrm{GaO}_{\mathrm{x}}$ top electrode. b) $J V$ curves acquired after retracting and re-contacting the ${ }^{t}$ Graphene-quartz.

With the aim of giving more insights into the transport mechanism, the $J V$ curves were fitted considering the well-known conduction mechanism models for nonlinear IV curves (thermionic emission, Schottky emission, space charge limited conduction, Poole-Frenkel emission, FowlerNordheim tunneling and trap assisted tunneling) ${ }^{29,30}$ For the HRS (from $0.2 \mathrm{~V}$ to $+1.5 \mathrm{~V}$ ) in the first sweeping cycle, the $J V$ curve was well fitted by the $\ln \left(\mathrm{J} / \mathrm{V}^{2}\right) \propto 1 / \mathrm{V}$ formula according to a FowlerNordheim tunneling (F-NT) (Figure S12). Although, there is a turning point voltage around $1 \mathrm{~V}$ which could be a signature of a transition from direct tunneling (DT) to F-NT, ${ }^{29}$ here it cannot be conclusively stated due to the fact of having an ill-defined top-electrode/substrate interface. ${ }^{31,32}$ This result points that an electrode-limited conduction mechanism and not bulk-limited is governing the charge transport across the $\mathrm{EGaIn} / \mathrm{GaOx} / \mathrm{tGr}-\mathrm{q}$ junction investigated in this work.

Motivated by the interesting electrical response observed in Figure 2b, the switching performance was investigated in detail. First, in order to read without destroying the state of the junction, $J V$ 
curves up to $|0.5 \mathrm{~V}|$ were acquired after applying a write/erase pulse of $+/-2.2 \mathrm{~V}$. The potential scan was performed from $0 \mathrm{~V}$ to +0.5 to $-0.5 \mathrm{~V}$ to $0 \mathrm{~V}$. As depicted in Figure 3a, the LRS was written with a pulse of $+2.2 \mathrm{~V}$ applied for 1 second, and then kept at $0 \mathrm{~V}$ for 10 seconds, leading to the HRS state (see heatmap of all data in Figure S13). Remarkably, the ON/OFF ratio at 0.5 V reached a value of $\sim 1 \times 10^{4}$, which is comparable with the values reported for gallium-based oxide conductivebridging RAM $^{18}$ or graphene oxide devices. ${ }^{7,33-35}$ In addition, the LRS and HRS were monitored every 500 ms by applying a read potential of 0.2 V. Importantly, as demonstrated in Figure 3b, the states were preserved for more than one hour. Figure 3c shows the distribution of the ON/OFF ratio for 36 junctions over 40 attempts, which clearly elucidates the reproducibility of the measurements and thus, of the in-situ generated resistive switching device. This was further supported by the observed reproducibility on the threshold $\left(\mathrm{V}_{\text {th }}\right)$ or turn ON/OFF voltages and the ON and OFF currents, as shown in the histograms in Figure S14.

In order to explore the robustness of the $\mathrm{EGaIn} / \mathrm{GaO}_{\mathrm{x}} /{ }^{t} \mathrm{Gr}-\mathrm{q}$ junctions, endurance switching measurements were performed. To achieve this purpose, pulses of $+2.2 \mathrm{~V}$ were applied for $1 \mathrm{~ms}$ then a potential was settled to $0.2 \mathrm{~V}$ to read the states. This read potential was kept for $1 \mathrm{~ms}$ and then the current was read using an integration time of 5ms. In this way 120 switches could be performed in a junction in less than 1s (Figure 3d). The red point indicates that the junction was broken after 120 switching process. Figure S15 shows the "offset" of the equipment during the measurement of endurance no contacting the sample. These values represent $\sim 4 \%$ of the measured values indicating the reliability of the measurements, especially in the LRS. The performance of the switch was also investigated under argon and oxygen enriched atmosphere and at high humidity (70\%) level since it is well known that water and oxygen can have a relevant impact on the measurements. ${ }^{36}$ The memory behavior was not achieved under argon (when the tip was also 
prepared under inert atmosphere), probably due to the poor oxide layer formed on the tip. On the contrary, the switching performance became more robust (enhanced cyclability and high reproducible $\mathrm{V}_{\text {th }}$ ) when the tip formation and the electrical measurements were performed under a flow of oxygen as shown in Figure S16. This iss attributed to the lower presence of oxide defects at the EGaIn surface (as mentioned before for the contact area experiments). In the case of the measurements performed increasing the humidity of the chamber, although preserving the switching function, some additional peaks around $+0.7 \mathrm{~V}$ appear, which are attributed to some redox reactions occurring at the interface. It has been observed that in air the LRS is not retained, with or without the tip retracting step. In contrast, when working in an oxygen rich atmosphere the LRS state is maintained even after 16 hours keeping the tip in contact (Figure S17).
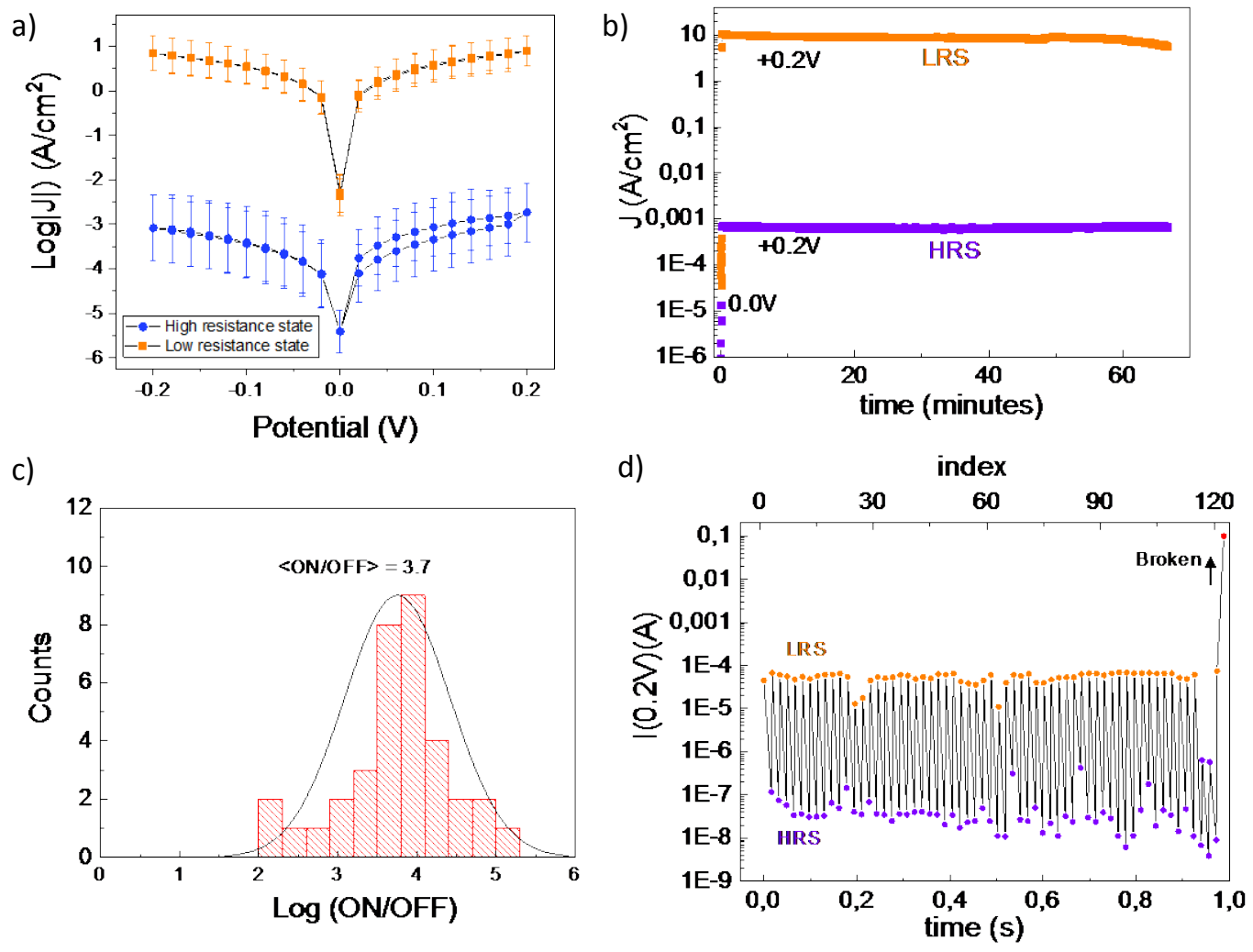
Figure 3. Measurements on a EGaIn/GaOx ${ }^{\prime t} \mathrm{Gr}$-quartz junction a) Average $J V$ curve with error bars of the reading process. b) $J$ vs time measurements to determine the retention of the LRS and HRS. c) Distribution of ON/OFF ratio. Yield of successful junction formation $=82 \%$. Mean value $=3.7$ \pm 0.8 . d) Current measured after ON or OFF state settle.

Regarding the governing switching mechanism, we should consider that, contrary to other reported oxide resistive switching structures, here, the LRS is achieved at positive voltage (about $+1.2 \mathrm{~V}$ ) and the HRS at negative bias, which is the opposite to what it would be expected if the main origin was the electrochemical modulation of the gallium oxide, with the subsequent metal valence change. ${ }^{17}$ Taking this into account, an electronic effect and not a filament based one, is proposed as possible origin of the switching. ${ }^{3}$

In order to gain insights into the origin of the switching behavior, the role of the substrate was interrogated. SEM images (Figure S18) of the ${ }^{t} \mathrm{Gr}$-q used in this work show that this has line defects and disruptions such as wrinkles, ripples and folding. ${ }^{37}$ Such imperfections have previously been shown to affect graphene transport properties through the scattering of the charge carriers. This potential formation of highly resistive grain boundaries could lead to the carriers being trapped periodically in domains. ${ }^{38}$ It is also worth mentioning that, as observed in field-effect transistors based on transferred graphene onto $\mathrm{SiO}_{2},{ }^{39}$ the charge accumulation at graphene/quartz interface and the detrapping (or charge compensation) under negative bias could take place. This is a contribution that cannot be dismissed in this work but it has not been experimentally proved.

In order to better understand the possible role of the non-conductive substrate underneath the graphene, cleaved highly oriented pyrolytic graphite (HOPG) and CVD grown graphene on $\mathrm{Cu}$ were used at ambient atmosphere. In the case of the HOPG contacted with the EGaIn/GaOx cone 
the hysteretic behavior was observed (in the $\pm 2 \mathrm{~V}$ voltage range, Figure S19) but it showed weak resistive switching ability since in most of the junctions failed giving short circuits. Further, CVD graphene grown on $\mathrm{Cu}$ as well as CVD graphene transferred onto Au did not lead the formation of the switching junction (Figure S20). This experiment was also designed to explore the role of possible PMMA residues (from the wet transfer, see XPS in Figure $\mathrm{S}^{40}$ ) on the top of graphene and/or other contaminants below the graphene layer $\left(\mathrm{H}_{2} \mathrm{O}\right.$ molecules for example) but, the conductive substrate dominates the electrical performance of the junction.

Taking all this into consideration, we suggest that the origin of the induced resistive switching relies on a charge trapping/detrapping phenomenon. ${ }^{41}$ We propose that a charge trapping layer is formed at the $\mathrm{GaOx} / \mathrm{Graphene}$ interface and, when the voltage applied is higher than $\sim+1.5 \mathrm{~V}$, the traps are filled and the effective tunnel barrier becomes shorter. Consequently, the current increases exponentially, i.e. similar to moving from a trap-limited to trap-free regime. The strong linearity in the low resistance state indicates that the tunnel barrier is highly transparent to the electron transport. When the voltage reaches $\sim-1.5 \mathrm{~V}$, the trapped charges are released and the effective tunnel barrier thickness increases with the concomitant reduction of the current. Our future efforts will be devoted to perform temperature dependent experiments and device modelling ${ }^{42}$ to deeply investigate the conduction and resistive switching mechanism.

\section{CONCLUSIONS}

In summary, it has been demonstrated that besides the tremendous advantage and opportunities that liquid metals bring for the design of new electronic devices, the measurement protocol is as important as the material to achieve high performance as well as reproducible and reliable results. The experimental approach reported here based on a liquid metal/liquid metal-oxide shaped as a cone hanging from a syringe is a very easy procedure to generate resistive-switching devices at 
specific and small locations on transferred CVD monolayer graphene, achieving an ON/OFF ratio between the HRS and LRS at $0.5 \mathrm{~V}$ of $\sim 1 \times 10^{4}$. The memory response from the $\mathrm{EGaIn} / \mathrm{GaO}_{\mathrm{x}}{ }^{\prime} \mathrm{Gr}-$ quartz junctions has been shown to be very robust and reproducible.

\section{ASSOCIATED CONTENT}

Supporting Information. The following files are available free of charge. A scheme of the home-made set-up employed for the electrical characterization. Additional JV curves and XPS characterization of the sample (PDF)

\section{AUTHOR INFORMATION}

\section{Corresponding Author}

*Núria Crivillers. Molecular Nanoscience and Organic Materials Department (ICMAB-CSIC). ORCID: 0000-0001-6538-2482; EMAIL: ncrivillers@icmab.es.

\section{Author Contributions}

NC and DG designed the experiment. DG and JAdS fabricated and characterized the devices. DG, JAdS, MM and NC discussed the results. The manuscript was written through contributions of all authors. All authors have given approval to the final version of the manuscript. $¥$ These authors contributed equally.

ACKNOWLEDGMENT 
This work was supported by MINECO through the "Severo Ochoa" Programme for Centers of Excellence in R\&D (SEV-2015-0496) and the FUNMAT-FIP projects. The authors also thank the CIBER-BBN, the Dirección General de Investigación (DGI) (Spain) project FANCY CTQ201680030-R and project GENESIS PID2019-111682RB-I00 and the Generalitat de Catalunya (2017SGR-918). JAdS is enrolled in the Materials Science doctoral program of the UAB. JAdS thanks the MINECO for the FPI-SO fellowship.

\section{REFERENCES}

(1) Li, Q.; Qiu, L.; Wei, X.; Dai, B.; Zeng, H. Point Contact Resistive Switching Memory Based on Self-Formed Interface of Al/ITO. Sci. Rep. 2016, 6, 29347.

(2) Zhang, S. R.; Zhou, L.; Mao, J. Y.; Ren, Y.; Yang, J. Q.; Yang, G. H.; Zhu, X.; Han, S. T.; Roy, V. A. L.; Zhou, Y. Artificial Synapse Emulated by Charge Trapping-Based Resistive Switching Device. Adv. Mater. Technol. 2019, 4, 1800342-1800348.

(3) Slesazeck, S.; Mikolajick, T. Nanoscale Resistive Switching Memory Devices: A Review. Nanotechnology 2019, 30, 352003.

(4) Ye, C.; Wu, J.; He, G.; Zhang, J.; Deng, T.; He, P.; Wang, H. Physical Mechanism and Performance Factors of Metal Oxide Based Resistive Switching Memory: A Review. J. Mater. Sci. Technol. 2016, 32, 1-11.

(5) Kim, S.; Jung, H. J.; Kim, J. C.; Lee, K. S.; Park, S. S.; Dravid, V. P.; He, K.; Jeong, H. Y. In Situ Observation of Resistive Switching in an Asymmetric Graphene Oxide Bilayer Structure. ACS Nano 2018, 12, 7335-7342.

(6) Liu, J.; Yin, Z.; Cao, X.; Zhao, F.; Wang, L.; Huang, W.; Zhang, H. Fabrication of Flexible, 
All-Reduced Graphene Oxide Non-Volatile Memory Devices. Adv. Mater. 2013, 25, 233238.

(7) Nagareddy, V. K.; Barnes, M. D.; Zipoli, F.; Lai, K. T.; Alexeev, A. M.; Craciun, M. F.; Wright, C. D. Multilevel Ultrafast Flexible Nanoscale Nonvolatile Hybrid Graphene Oxide-Titanium Oxide Memories. ACS Nano 2017, 11, 3010-3021.

(8) Porro, S.; Accornero, E.; Pirri, C. F.; Ricciardi, C. Memristive Devices Based on Graphene Oxide. Carbon 2015, 85, 383-396.

(9) Bo, G.; Ren, L.; Xu, X.; Du, Y.; Dou, S. Recent Progress on Liquid Metals and Their Applications. Advances in Physics: X. 2018, 412-442.

(10) Daeneke, T.; Khoshmanesh, K.; Mahmood, N.; De Castro, I. A.; Esrafilzadeh, D.; Barrow, S. J.; Dickey, M. D.; Kalantar-Zadeh, K. Liquid Metals: Fundamentals and Applications in Chemistry. Chem. Soc. Rev. 2018, 4073-4111.

(11) Kalantar-Zadeh, K.; Tang, J.; Daeneke, T.; O’Mullane, A. P.; Stewart, L. A.; Liu, J.; Majidi, C.; Ruoff, R. S.; Weiss, P. S.; Dickey, M. D. Emergence of Liquid Metals in Nanotechnology. ACS Nano. 2019, 13, 7388-7395.

(12) Ghasemian, M. B.; Mayyas, M.; Idrus-Saidi, S. A.; Jamal, M. A.; Yang, J.; Mofarah, S. S.; Adabifiroozjaei, E.; Tang, J.; Syed, N.; O’Mullane, A. P.; Daeneke, T.; Kalantar-Zadeh, K. Self-Limiting Galvanic Growth of $\mathrm{MnO}_{2}$ Monolayers on a Liquid Metal-Applied to Photocatalysis. Adv. Funct. Mater. 2019, 29, 1901649.

(13) Secor, E. B.; Cook, A. B.; Tabor, C. E.; Hersam, M. C. Wiring up Liquid Metal: Stable and 
Robust Electrical Contacts Enabled by Printable Graphene Inks. Adv. Electron. Mater. 2018, 4, 1700843-1700849.

(14) So, J. H.; Koo, H. J.; Dickey, M. D.; Velev, O. D. Ionic Current Rectification in Soft-Matter Diodes with Liquid-Metal Electrodes. Adv. Funct. Mater. 2012, 22, 625-631.

(15) Chen, X.; Hu, H.; Trasobares, J.; Nijhuis, C. A. Rectification Ratio and Tunneling Decay Coefficient Depend on the Contact Geometry Revealed by in Situ Imaging of the Formation of EGaIn Junctions. ACS Appl. Mater. Interfaces 2019, 11, 21018-21029.

(16) Farrell, Z. J.; Tabor, C. Control of Gallium Oxide Growth on Liquid Metal Eutectic Gallium/Indium Nanoparticles via Thiolation. Langmuir 2018, 34, 234-240.

(17) Aoki, Y.; Wiemann, C.; Feyer, V.; Kim, H.-S.; Schneider, C. M.; Ill-Yoo, H.; Martin, M. Bulk Mixed Ion Electron Conduction in Amorphous Gallium Oxide Causes Memristive Behaviour. Nat. Commun. 2014, 5, 3473-3481.

(18) Gan, K. J.; Liu, P. T.; Chien, T. C.; Ruan, D. B.; Sze, S. M. Highly Durable and Flexible Gallium-Based Oxide Conductive-Bridging Random Access Memory. Sci. Rep. 2019, 9, $14141-14147$.

(19) Ren, S.; Rong, P.; Yu, Q. Preparations, Properties and Applications of Graphene in Functional Devices: A Concise Review. Ceramics International. 2018, 11940-11955.

(20) Muñoz, R.; Gómez-Aleixandre, C. Review of CVD Synthesis of Graphene. Chem. Vap. Depos. 2013, 19, 297-322.

(21) Li, X.; Magnuson, C. W.; Venugopal, A.; Tromp, R. M.; Hannon, J. B.; Vogel, E. M.; 
Colombo, L.; Ruoff, R. S. Large-Area Graphene Single Crystals Grown by Low-Pressure Chemical Vapor Deposition of Methane on Copper. J. Am. Chem. Soc. 2011, 133, 28162819.

(22) Chua, C. K.; Pumera, M. Chemical Reduction of Graphene Oxide: A Synthetic Chemistry Viewpoint. Chem. Soc. Rev. 2014, 291-312.

(23) Boscá, A.; Pedrós, J.; Martínez, J.; Palacios, T.; Calle, F. Automatic Graphene Transfer System for Improved Material Quality and Efficiency. Sci. Rep. 2016, 6, 21676-21683.

(24) Magnuson, C. W.; Ruoff, R. S.; Goldberg, B. B.; Ahmed, S.; An, J.; Suk, J. W.; Kitt, A.; Hao, Y.; Swan, A. K. Transfer of CVD-Grown Monolayer Graphene onto Arbitrary Substrates. ACS Nano 2011, 5, 6916-6924.

(25) Stampfer, C.; Haupt, F.; Taniguchi, T.; Schmitz, M.; Banszerus, L.; Watanabe, K.; Oellers, M.; Dauber, J.; Engels, S.; Beschoten, B. Ultrahigh-Mobility Graphene Devices from Chemical Vapor Deposition on Reusable Copper. Sci. Adv. 2015, 1, e1500222.

(26) Xia, Z.; Leonardi, F.; Gobbi, M.; Liu, Y.; Bellani, V.; Liscio, A.; Kovtun, A.; Li, R.; Feng, X.; Orgiu, E.; Samorì, P.; Treossi, E.; Palermo, V. Electrochemical Functionalization of Graphene at the Nanoscale with Self-Assembling Diazonium Salts. ACS Nano 2016, 10, $7125-7134$.

(27) Chiechi, R. C.; Weiss, E. A.; Dickey, M. D.; Whitesides, G. M. Eutectic Gallium-Indium (EGaIn): A Moldable Liquid Metal for Electrical Characterization of Self-Assembled Monolayers. Angew. Chemie - Int. Ed. 2008, 47, 142-144. 
(28) Lesiak, B.; Kövér, L.; Tóth, J.; Zemek, J.; Jiricek, P.; Kromka, A.; Rangam, N. C Sp ${ }^{2} / \mathrm{Sp}^{3}$ Hybridisations in Carbon Nanomaterials - XPS and (X)AES Study. Appl. Surf. Sci. 2018, 452, 223-231.

(29) Yan, X.; Zhou, Z.; Ding, B.; Zhao, J.; Zhang, Y. Superior Resistive Switching Memory and Biological Synapse Properties Based on a Simple $\mathrm{TiN} / \mathrm{SiO}_{2} / \mathrm{p}-\mathrm{Si}$ Tunneling Junction Structure. J. Mater. Chem. C 2017, 5, 2259-2267.

(30) Chiu, F. C. A Review on Conduction Mechanisms in Dielectric Films. Adv. Mater. Sci. Eng. 2014, 2014, 1-18.

(31) Karuppannan, S. K.; Hongting, H.; Troadec, C.; Vilan, A.; Nijhuis, C. A. Ultrasmooth and Photoresist-Free Micropore-Based EGaIn Molecular Junctions: Fabrication and How Roughness Determines Voltage Response. Adv. Funct. Mater. 2019, 29, 1904452-190466.

(32) Ricœur, G.; Lenfant, S.; Guérin, D.; Vuillaume, D. Molecule/Electrode Interface Energetics in Molecular Junction: A “Transition Voltage Spectroscopy” Study. J. Phys. Chem. C 2012, 116, 20722-20730.

(33) Khurana, G.; Misra, P.; Katiyar, R. S. Forming Free Resistive Switching in Graphene Oxide Thin Film for Thermally Stable Nonvolatile Memory Applications. J. Appl. Phys. 2013, 114, 124508.

(34) Wang, L. H.; Yang, W.; Sun, Q. Q.; Zhou, P.; Lu, H. L.; Ding, S. J.; Wei Zhang, D. The Mechanism of the Asymmetric SET and RESET Speed of Graphene Oxide Based Flexible Resistive Switching Memories. Appl. Phys. Lett. 2012, 100, 63509. 
(35) Zhuge, F.; Hu, B.; He, C.; Zhou, X.; Liu, Z.; Li, R. W. Mechanism of Nonvolatile Resistive Switching in Graphene Oxide Thin Films. Carbon 2011, 49, 3796-3802.

(36) Wimbush, K. S.; Fratila, R. M.; Wang, D.; Qi, D.; Liang, C.; Yuan, L.; Yakovlev, N.; Loh, K. P.; Reinhoudt, D. N.; Velders, A. H.; Nijhuis, C. A. Bias Induced Transition from an Ohmic to a Non-Ohmic Interface in Supramolecular Tunneling Junctions with Ga2O3/EGaIn Top Electrodes. Nanoscale 2014, 6, 11246-11258.

(37) Park, K. S.; Kim, S.; Kim, H.; Kwon, D.; Koo Lee, Y. E.; Min, S. W.; Im, S.; Choi, H. J.; Lim, S.; Shin, H.; Koo, S. M.; Sung, M. M. Wafer-Scale Single-Domain-like Graphene by Defect-Selective Atomic Layer Deposition of Hexagonal ZnO. Nanoscale 2015, 7, 1770217709.

(38) Kholmanov, I. N.; Magnuson, C. W.; Aliev, A. E.; Li, H.; Zhang, B.; Suk, J. W.; Zhang, L. L.; Peng, E.; Mousavi, S. H.; Khanikaev, A. B.; Piner, R.; Shvets, G.; Ruoff, R. S. Improved Electrical Conductivity of Graphene Films Integrated with Metal Nanowires. Nano Lett. 2012, 12, 5679-5683.

(39) Lee, Y. G.; Kang, C. G.; Jung, U. J.; Kim, J. J.; Hwang, H. J.; Chung, H. J.; Seo, S.; Choi, R.; Lee, B. H. Fast Transient Charging at the Graphene/ $\mathrm{SiO}_{2}$ interface Causing Hysteretic Device Characteristics. Appl. Phys. Lett. 2011, 98, 44703.

(40) Christodoulou, C.; Giannakopoulos, A.; Nardi, M. V; Ligorio, G.; Oehzelt, M.; Chen, L.; Pasquali, L.; Timpel, M.; Giglia, A.; Nannarone, S.; Norman, P.; Linares, M.; Parvez, K.; Müllen, K.; Beljonne, D.; Koch, N. Tuning the Work Function of Graphene-on-Quartz with a High Weight Molecular Acceptor. J. Phys. Chem. C 2014, 118, 4784-4790. 
(41) Cui, Y.; Peng, H.; Wu, S.; Wang, R.; Wu, T. Complementary Charge Trapping and Ionic Migration in Resistive Switching of Rare-Earth Manganite TbMnO3. ACS Appl. Mater. Interfaces 2013, 5, 1213-1217.

(42) Sun, W.; Gao, B.; Chi, M.; Xia, Q.; Yang, J. J.; Qian, H.; Wu, H. Understanding Memristive Switching via in Situ Characterization and Device Modeling. Nature Commun. 2019, 10, 3453-3565.

TOC

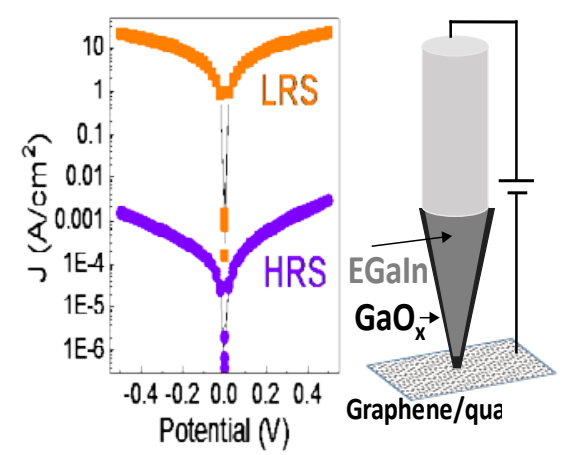

